\title{
Phonon transmission across an interface between two crystals
}

\author{
A. P. Meilakhs \\ Ioffe Physical Technical Institute, St. Petersburg, Russia \\ mejlaxs@mail.ioffe.ru
}

DOI 10.17586/2220-8054-2016-7-6-971-982

\begin{abstract}
A new model of phonon transmission across interface between two crystals is proposed which takes into account the mismatch of crystal lattices. It has been found that the mismatch of lattices results in phonon scattering at the interface even in the absence of defects. As it has been shown, at the normal incidence, longitudinally polarized phonons have much larger transmission coefficient than that of transversely polarized phonons, excluding special resonance cases. Allowance for this factor results in a calculated Kapitza resistance value that is approximately three times greater. For the quasi one-dimensional case, an exact solution has been obtained.
\end{abstract}

Keywords: Interface, Kapitza resistance, Lattice dynamics.

Received: 6 March 2016. Revised: 4 August 2016

\section{Introduction}

When heat flows through the boundary between two crystals, the temperature at the interface experiences a sharp jump. The proportionality coefficient between the heat flux and the temperature jump is known as the thermal boundary resistance or the Kapitza resistance. The Kapitza resistance is a crucial parameter for calculating the heat conductivity of nanocomposite materials. These materials are needed for the development of compact, inexpensive, easily-fabricated heat sinks for electronic devices, primarily for computer processors [1,2]. The study of thermal transmission across the crystal interface, on in its own, attracts the attention to the problem of phonon transmission across the crystal interface since, in the majority of cases, the phonons are responsible for the energy transfer across the interface. Additionally, several thermal devices, such as thermal transistors [3], thermal rectifiers [4] and thermal logical gates [5] have been recently suggested. An accurate description of crystal lattice dynamics is important for these purposes.

Various approximations are used in the theory of thermal boundary resistance. Two basic approaches are the so called Acoustic Mismatch Model (AMM) and Diffusive Mismatch Model (DMM). In the first approach [6,7], it is suggested that the interfacial coefficients of the phonon transmission and reflection could be calculated using the elasticity theory. However, firstly, this approximation is suitable only for calculation of the Kapitza resistance at low temperatures. Secondly, this approximation doesnt take into account the phonon scattering at the crystal interface. The values of the Kapitza resistance calculated within AMM occurred significantly higher than the experimentally determined values [8].

The second approach [9] suggests, vice versa, that the interfacial scattering is very strong, and phonons incident at the interface 'forget' their initial direction and uniformly scatter in all directions. The Kapitza resistance calculated within this approach is higher than the value calculated within the AMM, but still doesn't give the good agreement with the experimental data [8].

Thus, it is necessary to develop more accurate model of the phonon transmission across the interface, which takes into account the atomic structure of actual crystals. The simplest model of this kind is the one-dimensional chain with the interface. Such a model has been detailed in papers [10,11]. Paper [12] describes the threedimensional model of lattice dynamics near the interface. However, this model does not take into consideration the lattice mismatch of the two crystals.

More complicated models are studied by computer simulation. Thus, in paper [13] it was found that longitudinal phonons have the significantly higher interfacial transmittance coefficient than the transverse phonons. Phonon transmission across the interface is studied with a Green's function method in [14]. It is also suggested that atoms located at the interface are randomly displaced from the position that they would occupy if they were in volume of the crystal.

The role of anharmonicity of oscillations in the phonon transmission across the interface is considered in the paper [15]. In the most recent study [16], it has been found that atoms at the interface oscillate with a frequency exceeding the maximum possible frequency in a given crystal.

The presented paper proposes an analytical description of dynamics of the crystal lattice near the interface which takes into account the mutual mismatch of the lattices. The main assumption is that displacements of atoms at the interface are not random, but determined by forces affecting them from the side of atoms of the adjacent 
crystal. Generally the exact solution in such a model is not possible. At the same time, the essential qualitative effects related to the lattice mismatch can be found. In particular, it is possible to explain why the transverse phonons have much lower transmission coefficients compared to longitudinal ones. It turns out that the lattice mismatch, even in the absence of defects, results in phonon scattering at the interface. This scattering is not random, but has a certain structure.

\section{The model}

We consider the interface between two crystals having the structure of a simple cubic lattice and contacted by both crystal surfaces $(1,0,0)$ (Fig.1). The axis $x$ is normal to the interface. In each of the crystals we take into account the interaction with the atoms of the first and second coordination groups. Such a model of a three-dimensional crystal lattice is the most simple and well-studied [17].

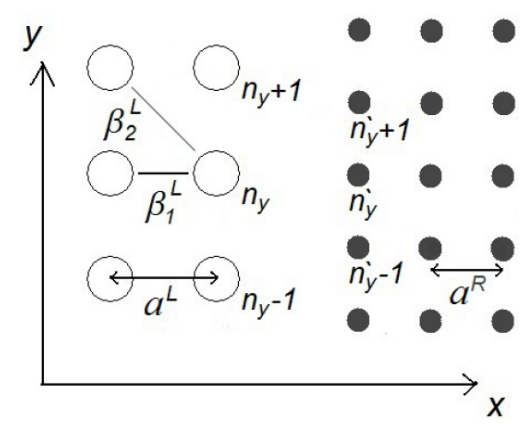

FIG. 1. Shown are the interface between two crystals (viewed from the side) and the atomic bonds considered in the model. The lattice constants are denoted as $a^{L}, a^{R}$; the interfacial atoms are numbered.

The lattice constants for the crystals on the left and on the right are $a^{L}, a^{R}$, respectively. The constants of the quasi elastic coupling with the atoms of the first and second coordination groups for the atoms of the left crystal and for the atoms of the right crystal are $\beta_{1}^{L}, \beta_{2}^{L}, \beta_{1}^{R}, \beta_{2}^{R}$, accordingly. We neglect the changes of the quasi elastic coupling constants and lattice constants near the interface. It is assumed that interactions between the atoms are due to short-range forces, and that with the atoms of the opposite crystal, only the atoms lying at the interface interact.

We introduce the following numbering: $n_{x}$ is the numbering of atoms along the axis $x$. For the left crystal the numbering goes from minus infinity to zero. Atoms in the interface plane are numbered as zeroes. For the right crystal the numbering goes from zero to plus infinity. $n_{y}, n_{z}$ is the numbering of atoms in the interface plane, it goes from the minus to plus infinity. The bold marking denotes a set of indexes $\mathbf{n}=\left(n_{x}, n_{y}, n_{z}\right)$.

Atoms located near the interface are influenced by an external potential produced by atoms from the opposite side of the interface. Due to this influence, the crystal is deformed, and the equilibrium position of an atom changes relative to its position in an ideal crystal. We perform the expansion of energy in the Taylor series in displacements of atoms from the positions which the nearest-to-interface atoms would occupy if they would not interact with atoms of an adjacent crystal. Derivatives of higher than third order, i.e. anharmonic terms, are not taken into consideration. The following expressions are valid for atoms on both sides therefore the index denoted the side is omitted.

Thus, we obtain:

$$
U\left(. ., \vec{r}_{\mathbf{n}}, \ldots\right)=\sum_{\mathbf{n}, \alpha} \frac{\partial U}{\partial r_{\mathbf{n}, \alpha}} r_{\mathbf{n}, \alpha}+\frac{1}{2} \sum_{\mathbf{n}, \alpha} \sum_{\mathbf{l}, \beta} \frac{\partial^{2} U}{\partial r_{\mathbf{n}, \alpha} \partial r_{\mathbf{l}, \beta}} r_{\mathbf{n}, \alpha} r_{\mathbf{l}, \beta},
$$

where $r_{\mathbf{n}, \alpha}$ - is the displacement of $\mathbf{n}$-th atom along the axis $\alpha, \alpha=x, y, z ; U\left(. ., \vec{r}_{\mathbf{n}}, \ldots\right)$ is the potential energy as a function of the displacement.

In the equilibrium position, the condition $\forall \mathbf{n}, \frac{\partial U}{\partial r_{\mathbf{n}, \alpha}}=0$ is fulfilled. We substitute the introduced expression for the potential energy into Eq. (1), and obtain:

$$
\frac{\partial U}{\partial r_{\mathbf{n}, \alpha}}+\sum_{\mathbf{l}, \beta} \frac{\partial^{2} U}{\partial r_{\mathbf{n}, \alpha} \partial r_{\mathbf{l}, \beta}} r_{\mathbf{l}, \beta}^{0}=0
$$


This system of $3 N$ equations ( $N$, number of atoms) determines the displacements $r_{1, \beta}^{0}$ at zero temperature in the absence of atomic oscillations, or 0-displacements, that are displacements of atoms from the position about which the potential energy is expanded into the Taylor series, to the equilibrium position.

Now, we set temperature to not equal to zero and atoms oscillate about the equilibrium position. Then the total displacement of an atom consists of the 0-displacement and its displacement due to thermal oscillations: $r_{\mathbf{n}, \alpha}=r_{\mathbf{n}, \alpha}^{0}+u_{\mathbf{n}, \alpha}$. The expression for the potential energy can be rewritten as:

$$
U\left(. ., \vec{r}_{\mathbf{n}}, \ldots\right)=\sum_{\mathbf{n}, \alpha} \frac{\partial U}{\partial r_{\mathbf{n}, \alpha}}\left(r_{\mathbf{n}, \alpha}^{0}+u_{\mathbf{n}, \alpha}\right)+\frac{1}{2} \sum_{\mathbf{n}, \alpha} \sum_{\mathbf{l}, \beta} \frac{\partial^{2} U}{\partial r_{\mathbf{n}, \alpha} \partial r_{\mathbf{l}, \beta}}\left(r_{\mathbf{n}, \alpha}^{0}+u_{\mathbf{n}, \alpha}\right)\left(r_{\mathbf{l}, \beta}^{0}+u_{\mathbf{l}, \beta}\right) .
$$

According to the Newtons second law:

$$
m \ddot{u}_{\mathbf{n}, \alpha}=-\frac{\partial U}{\partial u_{\mathbf{n}, \alpha}}=-\frac{\partial U}{\partial r_{\mathbf{n}, \alpha}}-\sum_{\mathbf{l}, \beta} \frac{\partial^{2} U}{\partial r_{\mathbf{n}, \alpha} \partial r_{\mathbf{l}, \beta}}\left(r_{\mathbf{l}, \beta}^{0}+u_{\mathbf{l}, \beta}\right) .
$$

By using the definition of the 0-displacement (2), we have:

$$
m \ddot{u}_{\mathbf{n}, \alpha}=-\sum_{\mathbf{l}, \beta} \frac{\partial^{2} U}{\partial r_{\mathbf{n}, \alpha} \partial r_{\mathbf{l}, \beta}} u_{\mathbf{l}, \beta} .
$$

Thus, by taking into account only the terms of the Taylor series containing derivatives of not higher than the second order, the 0-displacements are completely excluded from the equations of lattice vibrations.

Let us define the interface, at which the average 0-displacements are much less than the lattice constant, as ideal. Interaction of 0-displacements with lattice vibrations is revealed only if the terms of Taylor series with third and higher derivatives are taken into account. Obviously, if the model of an ideal interface could be described properly, the real interface, the latter should be sufficiently smooth, and the interaction between atoms on opposite sides of the interface essentially weaker than interaction between atoms of the same crystal.

\section{Equation for the quasi one-dimensional case}

Let's consider first the simple case where the phonon falls normally at the interface, that is, the wave vector components are parallel to the interface $q_{y}, q_{z}=0$.

We define $u_{\mathbf{n}, \alpha}^{L}$ to be the displacement of the $\mathbf{n}$-th atom lying on the left side of the interface along the axis $\alpha$, and $u_{\mathbf{n}^{\prime}, \beta}^{R}$ the displacement of the $\mathbf{n}^{\prime}$-th atom on the right side of the interface along the axis $\beta$. Newtons second law for the l-th atom of the left crystal lying at the interface is then given by:

$$
m \ddot{u}_{\mathbf{l}, \alpha}^{L}=-\sum_{\mathbf{n} \neq \mathbf{l}, \beta} \frac{\partial^{2} U}{\partial u_{\mathbf{l}, \alpha}^{L} \partial u_{\mathbf{n}, \beta}^{L}} u_{\mathbf{l}, \beta}^{L}-\sum_{\beta} \frac{\partial^{2} U}{\partial u_{\mathbf{l}, \alpha}^{L} \partial u_{\mathbf{l}, \beta}^{L}} u_{\mathbf{l}, \beta}^{L}-\sum_{\mathbf{n}^{\prime}, \beta} \frac{\partial^{2} U}{\partial u_{\mathbf{l}, \alpha}^{L} \partial u_{\mathbf{n}^{\prime}, \beta}^{R}} u_{\mathbf{n}^{\prime}, \beta}^{R} .
$$

We take into account that:

$$
\frac{\partial^{2} U}{\partial u_{\mathbf{l}, \alpha}^{L} \partial u_{\mathbf{1}, \beta}^{L}}=-\sum_{\mathbf{n} \neq \mathbf{l}} \frac{\partial^{2} U}{\partial u_{\mathbf{l}, \alpha}^{L} \partial u_{\mathbf{n}, \beta}^{L}}-\sum_{\mathbf{n}^{\prime}} \frac{\partial^{2} U}{\partial u_{\mathbf{l}, \alpha}^{L} \partial u_{\mathbf{n}^{\prime}, \beta}^{R}},
$$

as follows from the fact that the energy does not change if the atoms of both crystals are displaced by an equal distance in the same direction. Substitution of Eq. (7) in Eq. (6) gives:

$$
m \ddot{u}_{\mathbf{l}, \alpha}^{L}=-\sum_{\mathbf{n} \neq \mathbf{l}, \beta} \frac{\partial^{2} U}{\partial u_{\mathbf{l}, \alpha}^{L} \partial u_{\mathbf{n}, \beta}^{L}}\left(u_{\mathbf{n}, \beta}^{L}-u_{\mathbf{l}, \beta}^{L}\right)-\sum_{\mathbf{n}^{\prime}, \beta} \frac{\partial^{2} U}{\partial u_{\mathbf{l}, \alpha}^{L} \partial u_{\mathbf{n}^{\prime}, \beta}^{R}}\left(u_{\mathbf{n}^{\prime}, \beta}^{R}-u_{\mathbf{l}, \beta}^{L}\right) .
$$

The first term in the right part of Eq. (8) describes the interaction of an atom with atoms of the same crystal, the second one relates to the interaction with atoms lying on the other side of the interface.

We seek a solution in the form of superposition of the incident, reflected and transmitted waves. We do not take into consideration the wave scattering at the interface, so that the wave vector components of the reflected and transmitted waves, parallel to the interface, equal zero. In this case, there are three reflected and three transmitted waves with different polarizations. Let us assume that from the left, at the interface falls the wave of unit amplitude and polarization 1. Then, for an atom on the left side (not necessary at the interface itself) we obtain:

$u_{\mathbf{n}, \alpha}^{L}=\exp (i \omega t)\left(\exp \left(-i q_{1}^{L} a^{L} n_{x}\right) e_{1 \alpha}^{L}+A_{1} \exp \left(i q_{1}^{L} a^{L} n_{x}\right) e_{1 \alpha}^{L}+A_{2} \exp \left(i q_{2}^{L} a^{L} n_{x}\right) e_{2 \alpha}^{L}+A_{3} \exp \left(i q_{3}^{L} a^{L} n_{x}\right) e_{3 \alpha}^{L}\right)$.

Here, indexes 1,2,3 denote polarization, $A_{1,2,3}$ amplitudes of the reflected waves with different polarization, $q_{1,2,3}$ are $x$-components of the wave vectors. $\vec{e}_{1,2,3}^{L}$ stand for the polarization vectors, $e_{\alpha}$ for the component of the 
polarization vector in direction of the axis $\alpha$. At the interface $n_{x}=0$, so for interfacial atoms each exponent in Eq. (9) becomes unity.

The wave vector values $q_{1,2,3}$ are assumed to be known for the given frequency $\omega$, because the disperse relation for the waves in a simple cubic lattice is known [17]. Near the interface, it also may be that the values $q_{2,3}^{L}$ are imaginary, that is, the oscillation is gradually damping inward into the crystal (see the paper [11] and discussion in section 7). Subsequently, we assume that the phonon falls at the interface from the left, and so the crystal from the side of which the phonon falls, will be called as 'left' for brevity.

Similarly, for crystal atoms to the right of the interface we have:

$$
u_{\mathbf{n}^{\prime}, \alpha}^{R}=\exp (i \omega t)\left(B_{1} \exp \left(-i q_{1}^{R} a^{R} n_{x}^{\prime}\right) e_{1 \alpha}^{R}+B_{2} \exp \left(-i q_{2}^{R} a^{R} n_{x}^{\prime}\right) e_{2 \alpha}^{R}+B_{3} \exp \left(-i q_{3}^{R} a^{R} n_{x}^{\prime}\right) e_{3 \alpha}^{R}\right),
$$

where $B_{1,2,3}$ are the amplitudes of the transmitted waves.

Equations (9) and (10) are the solutions to Newtons equations for the atoms lying off the interface, since $q_{1,2,3}$ and $\omega$ satisfy the dispersion relations for lattice vibrations. The problem thus reduces to finding the $A_{1,2,3}, B_{1,2,3}$ which would satisfy Newtons equations for the interfacial atoms. For this purpose we substitute Eqs. (9) and (10) in Eq. (8) and try to simplify the expression obtained. For the term describing the interaction of an atom with atoms of the same crystal, we can proceed in a way similar to that in [12]. We divide both parts of Eq. (8) by $\exp (i \omega t)$, and then for the first term in the right part, we have:

$$
\begin{array}{r}
\sum_{\mathbf{n} \neq \mathbf{l}, \beta} \frac{\partial^{2} U}{\partial u_{\mathbf{l}, \alpha}^{L} \partial u_{\mathbf{n}, \beta}^{L}}\left(u_{\mathbf{n}, \beta}^{L}-u_{\mathbf{l}, \beta}^{L}\right)=\sum_{\mathbf{n} \neq \mathbf{l}, \beta} \frac{\partial^{2} U}{\partial u_{\mathbf{l}, \alpha}^{L} \partial u_{\mathbf{n}, \beta}^{L}}\left(\left(1-\exp \left(-i q_{1}^{L} a^{L} n_{x}\right)\right) e_{1 \beta}^{L}+\sum_{j} A_{j}\left(1-\exp \left(i q_{j}^{L} a^{L} n_{x}\right)\right) e_{j \beta}^{L}\right)= \\
\sum_{\mathbf{n} \neq l, \beta} \frac{\partial^{2} U}{\partial u_{\mathbf{l}, \alpha}^{L} \partial u_{\mathbf{n}, \beta}^{L}}\left(1-\exp \left(-i q_{1}^{L} a^{L} n_{x}\right)\right) e_{1 \beta}^{L}++\sum_{j} A_{j} \sum_{\mathbf{n} \neq \mathbf{l}, \beta} \frac{\partial^{2} U}{\partial u_{\mathbf{l}, \alpha}^{L} \partial u_{\mathbf{n}, \beta}^{L}}\left(1-\exp \left(-i q_{j}^{L} a^{L} n_{x}\right)\right) e_{j \beta}^{L} .
\end{array}
$$

We take into account that the frequency and the polarization vector can be expressed in terms of the dynamic matrix [17], for both crystals:

$$
\omega^{2} e_{j \alpha}=\frac{1}{m} \sum_{\mathbf{n} \neq \mathbf{l}, \beta} \frac{\partial^{2} U}{\partial u_{\mathbf{l}, \alpha} \partial u_{\mathbf{n}, \beta}}\left(1-\exp \left(-i q_{j} a\left(n_{x}-l_{x}\right)\right) e_{j \beta}=\sum_{\beta} D_{\alpha \beta}\left(q_{j}\right) e_{j \beta}\left(q_{j}\right) .\right.
$$

In Eq. (11), the summation is over the atoms of the same crystal only. To the right of the interfacial atom of the left crystal (for interfacial atoms $n_{x}=0$ ), there are no more atoms of that crystal $\left(n_{x} \leq 0\right)$, that can be formally taken into consideration in the form of the condition $l_{x} \leq n_{x}$. We introduce the notation:

$$
D_{\alpha \beta}^{L \times}\left(q_{j}\right)=\frac{1}{m^{L}} \sum_{\substack{\mathbf{l} \neq \mathbf{n}, \beta \\ l_{x} \leq n_{x}}} \frac{\partial^{2} U}{\partial u_{\mathbf{n}, \alpha}^{L} \partial u_{\mathbf{l}, \beta}^{L}}\left(1-\exp \left(i q_{j}^{L} a^{L} l_{x}\right)\right)
$$

which is for the dynamic matrix describing the atom lying at the interface. Here, we can ignore the fact that $n_{x} \leq 0$, since for an interfacial atom it was taken into account in the expression $l_{x} \leq n_{x}$. So we can operate with Eq. (13) formally.

Denote the difference of the two dynamic matrices (one for an atom in the depth of the crystal and the other for an atom at the interface) as:

$$
{ }^{\otimes} D_{\alpha \beta}^{L}\left(q_{j}^{L}\right)=D_{\alpha \beta}^{L}\left(q_{j}^{L}\right)-D_{\alpha \beta}^{L \times}\left(q_{j}\right)=\frac{1}{m^{L}} \sum_{\substack{\mathbf{1} \neq \mathbf{n}, \beta \\ l_{x}>n_{x}}} \frac{\partial^{2} U}{\partial u_{\mathbf{n}, \alpha}^{L} \partial u_{\mathbf{l}, \beta}^{L}}\left(1-\exp \left(i q_{j}^{L} a^{L} l_{x}\right)\right) .
$$

We substitute Eqs. (12), (13) in Eq. (8) and transfer the first term of the right part of Eq. (8) to the left. The expression thus obtained can be written in new notations in the form:

$$
\sum_{j} A_{j} \sum_{\beta}^{\otimes} D_{\alpha \beta}^{L}\left(q_{j}^{L}\right) e_{j \beta}^{L}+{ }^{\otimes} D_{\alpha \beta}^{L}\left(-q_{1}^{L}\right) e_{1 \beta}^{L}=-\sum_{\mathbf{n}^{\prime}, \beta} \frac{\partial^{2} U}{\partial u_{\mathbf{n}, \alpha}^{L} \partial u_{\mathbf{n}^{\prime}, \beta}^{R}}\left(u_{\mathbf{n}^{\prime}, \beta}^{R}-u_{\mathbf{n}, \beta}^{L}\right) .
$$

Now, we rearrange the right part of Eq.(15) by using the relation:

$$
K_{\mathbf{n}, \alpha \beta}=\sum_{\mathbf{n}^{\prime}} \frac{\partial^{2} U}{\partial u_{\mathbf{n}, \alpha}^{L} \partial u_{\mathbf{n}^{\prime}, \beta}^{R}},
$$

which is the matrix of the interfacial interaction, describing the interaction of an atom with atoms lying on the opposite side of the interface, where $n_{x}=0$ is supposed. Unlike the conventional dynamic matrix, this type of 
matrix depends on the $n$, because each atom at the interface is differently located relative to atoms on the opposite side of the interface.

Substituting Eqs. (10), (16) into Eq. (15), we come to:

$$
\sum_{j} A_{j} \sum_{\beta}^{\otimes} D_{\alpha \beta}^{L}\left(q_{j}^{L}\right) e_{j \beta}^{L}+{ }^{\otimes} D_{\alpha \beta}^{L}\left(-q_{1}^{L}\right) e_{1 \beta}^{L}=-\sum_{\beta} K_{\mathbf{n}, \alpha \beta}\left(-e_{1 \beta}^{L}+\sum_{j}\left(B_{j} e_{j \beta}^{R}-A_{j} e_{j \beta}^{L}\right)\right) .
$$

Then, we sum over all values of $n$ in Eq.(17) and divide the result by a total number of atoms $N$ at the interface on the left. The left part of the expression is unchanged for it is independent of the number $n$ of the atom. In the right part of the expression, we obtain:

$$
K_{\alpha \beta}^{L}=\frac{1}{N} \sum_{\mathbf{n}} K_{\mathbf{n}, \alpha \beta}
$$

This is the averaged matrix describing the interaction of atoms across the interface. By performing such an averaging, we do not take into account that the atoms nearest to the interface differ in their position relative to atoms on the opposite side of the interface, and, hence, interact differently with them. Taking account of this difference involves the appearance of scattering, which calls for mathematical technique presented below in Sec. 5 . In this section, we proceed ignoring the scattering. The problem in this case becomes quasi-one-dimensional.

Let the relation:

$$
M_{\alpha j}^{L, \pm}=\sum_{\beta}\left({ }^{\otimes} D_{\alpha \beta}^{L}\left( \pm q_{j}\right)-K_{\alpha \beta}^{L}\right) e_{j \beta}^{L},
$$

be the second dynamical matrix. It expresses the forces acting on atoms near the interface in terms of amplitudes of waves differing in polarization on the left side of the interface. To write the analogous expression for the forces acting on atoms on the other side of the interface, we introduce:

$$
I_{\alpha j}^{L}=\sum_{\beta} K_{\alpha \beta}^{L} e_{j \beta}^{R} .
$$

With new notations, Eq. (17) can be rewritten in the form:

$$
M_{\alpha j}^{L,-} A_{j}+I_{\alpha j}^{L} B_{j}=-M_{\alpha 1}^{L,+}
$$

where the summation is supposed to be performed over the repetitive index $j$ standing for the wave polarization.

By writing Newtons second law for atoms on the right of the interface, one can rearrange the obtained expression in a similar way. A distinction is in the fact that the averaged matrix of the interfacial interaction is defined as:

$$
K_{\beta \alpha}^{R}=\frac{1}{N^{\prime}} \sum_{\mathbf{n}^{\prime}} \sum_{\mathbf{n}} \frac{\partial^{2} U}{\partial u_{\mathbf{n}, \alpha}^{L} \partial u_{\mathbf{n}^{\prime}, \beta}^{R}}=\frac{N}{N^{\prime}} K_{\alpha \beta}^{L},
$$

where $N^{\prime}$ is a number of atoms nearest to the interface in the crystal on the right. The amounts of atoms near the interface are related as inverse squares of the lattice constants. Thus, we obtain:

$$
K_{\beta \alpha}^{R}=\left(\frac{a^{L}}{a^{R}}\right)^{2} K_{\alpha \beta}^{L},
$$

which represents the fact that the force acting on the right crystal from the side of the left one is equal, in magnitude, to the force acting on the left crystal from the side of the right one. Finally, we have:

$$
M_{\alpha j}^{L,-} A_{j}+I_{\alpha j}^{L} B_{j}=-M_{\alpha 1}^{L,+}, \quad M_{\alpha j}^{R,+} B_{j}+I_{\alpha j}^{R} A_{j}=-I_{\alpha 1}^{R} .
$$

This is the sought-for system of equations.

\section{Theorem on the interfacial interaction and the exact solution in the quasi-one-dimensional case}

Let us study the matrix of interfacial interaction, $K_{\alpha \beta}$, using the fact that the interaction energy of two crystals is invariant with respect to the displacements by any linear combination of the lattice vectors. We consider first the general case when the lattice parameters are incommensurate, that is, their ratio is irrational, $a^{L} / a^{R} \notin \mathbb{Q}$. In this case, linear combinations of the lattice vectors and, hence, a set of rearrangements converting the system into itself, is dense on the plane. It is reasonable to require that the energy be continuous as the function of the relative displacement of the crystals. Since the energy is constant on the dense set and, besides, is continuous, it is constant.

To be specific, we will demonstrate that $K_{y y}=0$. We displace the first crystal by a small distance $Y$ along the axis $y$. The energy in a new state will, on the one hand, be equal to the initial one, and on the other, be a different amount of work done by the force of interaction between crystals: 


$$
E=E+\int_{0}^{Y} K_{y y} y d y \Rightarrow K_{y y} Y^{2} / 2=0 \Rightarrow K_{y y}=0 .
$$

For the other seven components of the matrix $K_{\alpha \beta}$ (except for $K_{x x}$ ), the proof can be performed in a similar way. Thus, we conclude that, with initial assumptions, the only nonzero component of the matrix of interfacial interaction is $K_{x x}$.

We now consider a case of resonance where $a^{L} / a^{R} \in \mathbb{Q}$. It is obvious that the above reasoning is inapplicable. Let $a^{L} / a^{R}=p / q$ and $p / q<1$. The interaction energy $U$ of two crystals will be described by a periodical function of their relative displacement with a period $q a^{L}$. We expand it and $K_{\alpha \beta}$ into the Fourier series and substitute the result in Eq. (16). The first, largest term of the series turns out to be:

$$
K_{\alpha \beta 1}^{R}=\frac{1}{q^{2}\left(a^{L}\right)^{2}} U_{1} .
$$

We drop the other terms and obtain the following assessment for the case of equal potentials of the interatomic interaction:

$$
K_{\alpha \beta} \sim 1 / q^{2}, \quad a^{L} / a^{R}=p / q, \quad K_{\alpha \beta}=0, \quad a^{L} / a^{R} \notin \mathbb{Q} .
$$

Such a function experiences a discontinuity in all rational points, which is impossible from the physical point of view. This paradox can be resolved from the consideration that this function was obtained ignoring the interaction with 0 -displacements which are large near the resonance. Accounting for the anharmonic coupling with 0 -displacements leads to smearing of spikes at rational points. As a result, the actual matrix of the interfacial interaction will be given by a smooth envelope for the function (27).

Hence, we come to the following qualitative result: the atomic oscillations perpendicular to the plane of the interface interact intensively with the atomic oscillations on the opposite side of the interface only in the case when the ratio of the lattice constants is close to a rational number with a small denominator. Otherwise, this interaction is weak.

This result can be illustrated by an interesting analogy in nebular mechanics, where the close commensurability of revolution periods of planets, i.e., the proximity of a ratio of periods to a rational number with a small denominator, leads to the strong long-period perturbation [18].

Using the predetermined theorem on the matrix of the interfacial interaction, one can obtain an exact solution in the quasi-one-dimensional nonresonance case. We write explicitly the expressions for the elements of the matrix ${ }^{\otimes} D_{\alpha \beta}\left(q_{j}\right)$, recalling that in considered model we take into account the interaction with the atoms of the first and second coordination groups only (see Fig. 1), indexes $L, R$ are ommited:

$$
\begin{array}{r}
{ }^{\otimes} D_{x x}\left(q_{j}\right)=\frac{\beta_{1}+2 \beta_{2}}{m}\left(1-e^{i q_{x} a}\right), \\
{ }^{\otimes} D_{y y}\left(q_{j}\right)={ }^{\otimes} D_{z z}\left(q_{j}\right)=\frac{\beta_{2}}{m}\left(1-e^{i q_{x} a}\right), \\
{ }^{\otimes} D_{x y}\left(q_{j}\right)={ }^{\otimes} D_{x z}\left(q_{j}\right)={ }^{\otimes} D_{y z}\left(q_{j}\right)=0 .
\end{array}
$$

The remaining three components are also equal zero since the matrix is symmetrical.

We denote $K_{x x}^{L}=\beta^{L}, K_{x x}^{R}=\beta^{R}$.

Let a longitudinally polarized wave is incident at the interface. Substituting Eqs. (27), (28) in Eq. (18), we get:

$$
M_{x, 1}^{L, \pm}=\frac{\beta_{1}^{L}+2 \beta_{2}^{L}}{m^{L}}\left(1-\exp \left( \pm i q_{x}^{L} a^{L}\right)\right)-\frac{\beta^{L}}{m^{L}} .
$$

In addition, one can easily see that according to the theorem on the matrix of interfacial interaction all nondiagonal components of the matrix $M$ equal zero. Hence, when the incident wave is longitudinally polarized, the reflected and transmitted waves also have longitudinal polarization. The system of six linear equations (23) transforms into the simple system of two linear equations:

$$
M_{x, 1}^{L,-} A_{1}+\beta^{L} B_{1}=-M_{x, 1}^{L,+}, \quad M_{x, 1}^{R,+} B_{1}+\beta^{R} A_{1}=-\beta^{R},
$$

From whence, it follows:

$$
A_{1}=\frac{\beta^{L} \beta^{R}-M_{x, 1}^{L,+} M_{x, 1}^{R,+}}{M_{x, 1}^{L,-} M_{x, 1}^{R,+}-\beta^{L} \beta^{R}}, \quad B_{1}=\frac{\beta^{R}\left(M_{x, 1}^{L,+}-M_{x, 1}^{L,-}\right)}{M_{x, 1}^{R,+} M_{x, 1}^{L,-}-\beta^{L} \beta^{R}} .
$$

This solution coincides with the result obtained in the paper [10] for a one-dimensional chain if one rewrite it in the above notations. 
Later, we consider the case where the wave with transverse polarization is incident at the interface. For the sake of specificity, we assume that the oscillations occur along the axis $y$. Since, according to the theorem on the matrix of interfacial interaction we have $K_{y y}=0$, the corresponding equations for the transmission coefficient have the form:

$$
M_{y, 2}^{L,-} A_{2}=-M_{y, 1}^{L,+}, \quad M_{y, 2}^{R,+} B_{2}=0,
$$

whence $B_{2}=0,\left|A_{2}\right|=1$.

Actually, however, $K_{y y}$ is small but does not equal zero, because the 0-displacements, although small, are nonzero. It was shown in the paper [10] that for small incident wave frequencies, the transmission coefficient is independent of the interaction force of atoms at the interface, but determined by acoustic impedances of media on opposite sides of the interface. However, when the interaction force of interfacial atoms is weak, the transmission coefficient of phonons decreases fast with increased frequency. Since the heat transport is carried out by phonons of all frequencies, we can neglect the contribution to the energy transport across the interface from atomic oscillations parallel to the interface.

\section{Fourier transform of the matrix of interfacial interaction}

In order to study the phonon transmission across the interface between two crystals in more general case when a phonon is incident at arbitrary angle, and take into consideration the scattering, a special mathematical apparatus is required which is presented below. For simplicity, we consider the one-dimensional case, because subsequent generalization to more dimensions is trivial.

Let us have an infinite one-dimensional chain of atoms spaced $a^{L}$ apart. The atoms of the chain are numbered from minus infinity to plus infinity, and the axis $x$ is located so that the zero-th atom has the coordinate $x=0$. We place the chain into the external potential $\Phi(x)$ with a period $a^{R}, \Phi\left(x+a^{R}\right)=\Phi(x)$.

Let's expand the $\Phi(x)$ into the Fourier series:

$$
\begin{aligned}
\Phi_{k} & =\frac{1}{2 \pi a^{R}} \int_{0}^{a^{R}} \Phi(x) \exp \left(-2 \pi i k x / a^{R}\right) d x, \\
\Phi(x) & =\sum_{k=-\infty}^{+\infty} \Phi_{k} \exp \left(2 \pi i k x / a^{R}\right) .
\end{aligned}
$$

Then, the potential energy of $n$-th atom, $\Phi_{n}$, will equal $\Phi\left(n a^{L}\right)$, or

$$
\Phi_{n}=\sum_{k=-\infty}^{+\infty} \Phi_{k} \exp \left(2 \pi i k n\left(a^{L} / a^{R}\right)\right) .
$$

One can demonstrate that the inverse rearrangement is also valid:

$$
\Phi_{k}=\lim _{N \rightarrow \infty} \frac{1}{2 N} \sum_{n=-N}^{N} \Phi_{n} \exp \left(-2 \pi i k n\left(a^{L} / a^{R}\right)\right) .
$$

Actually,

$$
\begin{array}{r}
\lim _{N \rightarrow \infty} \frac{1}{2 N} \sum_{n=-N}^{N} \Phi_{n} \exp \left(-2 \pi i k n\left(a^{L} / a^{R}\right)\right) \\
=\lim _{N \rightarrow \infty} \frac{1}{2 N} \sum_{n=-N}^{N}\left(\sum_{k^{\prime}=-\infty}^{+\infty} \Phi_{k^{\prime}} \exp \left(2 \pi i k^{\prime} n\left(a^{L} / a^{R}\right)\right)\right) \exp \left(-2 \pi i k n\left(a^{L} / a^{R}\right)\right) \\
=\sum_{k^{\prime}=-\infty}^{+\infty} \Phi_{k^{\prime}} \lim _{N \rightarrow \infty} \frac{1}{2 N} \sum_{n=-N}^{N} \exp \left(2 \pi i\left(k^{\prime}-k\right) n\left(a^{L} / a^{R}\right)\right)=\sum_{k^{\prime}=-\infty}^{+\infty} \Phi_{k^{\prime}} \delta_{k^{\prime} k}=\Phi_{k} .
\end{array}
$$

For what follows, Eq. (34) can be conveniently rewritten so that it would correspond to the expansion in exponents with the wave vectors $q_{k}$ lying in the first Brillouin zone, i.e., $q_{k} \in\left(-\pi / a^{L}, \pi / a^{L}\right)$. To do so, we introduce:

$$
q_{k}=\frac{2 \pi}{a^{L}}\left(\left\{k \frac{a^{L}}{a^{R}}+\frac{1}{2}\right\}-\frac{1}{2}\right),
$$


and, finally, we obtain:

$$
\Phi_{n}=\sum_{k=-\infty}^{+\infty} \Phi_{k} \exp \left(i q_{k} a^{L} n\right) .
$$

Equation (34) can be applied to the matrix $K_{\mathbf{n}, \alpha \beta}$ and that to use it for description of the phonon transmission across the interface of two crystal we have to rearrange it. Really,

$$
K_{\mathbf{n}, \alpha \beta}=\frac{\partial}{\partial u_{\mathbf{n}, \alpha}^{L}} \sum_{\mathbf{n}^{\prime}} \frac{\partial U}{\partial u_{\mathbf{n}^{\prime}, \beta}^{R}} .
$$

The function:

$$
\Phi=\sum_{\mathbf{n}^{\prime}} \frac{\partial U}{\partial u_{\mathbf{n}^{\prime}, \beta}^{R}}
$$

is periodical (the period $a^{R}$ ), since the potential produced by atoms of the right crystal for atoms of the left crystal is periodical. Hence, we can introduce:

$$
\begin{array}{r}
K_{\mathbf{k}, \alpha \beta}=\lim _{N \rightarrow \infty} \frac{1}{N} \sum_{\mathbf{n}} K_{\mathbf{n}, \alpha \beta} \exp \left(-2 \pi i \mathbf{k n}\left(a^{L} / a^{R}\right)\right), \\
K_{\mathbf{n}, \alpha \beta}=\sum_{\mathbf{k}=-\infty}^{+\infty} K_{\mathbf{k}, \alpha \beta} \exp \left(2 \pi i \mathbf{k n}\left(a^{L} / a^{R}\right)\right),
\end{array}
$$

where $\mathbf{k}=\left(0, k_{y}, k_{z}\right)$.

\section{Equation and the theorem on the interfacial interaction in the general case}

Let the wave of the unit amplitude, polarization 1 and wave vector $\mathbf{q}_{1}$ fall at the interface. Notations: $\mathbf{q}_{\|}=\left(0, q_{y}, q_{z}\right)$ are the wave vector components parallel to the interface, and $\mathbf{n}_{\|}=\left(0, n_{y}, n_{z}\right)$ are the atom numbers along the axes $y, z$ parallel to the plane of the interface. We seek the solution for the left side in the form of superposition of the incident and reflected waves:

$$
\begin{array}{r}
u_{\mathbf{n}, \alpha}^{L}=\exp (i \omega t)\left(\exp \left(-i q_{1, x}^{L} n_{x} a^{L}+i \mathbf{q}_{\|}^{L} \mathbf{n}_{\|} a^{L}\right) e_{1 \alpha}^{L}\right. \\
\left.+\sum_{j, \mathbf{k}} A_{\mathbf{k}, j} \exp \left(i q_{j, x}^{L} n_{x} a^{L}+i\left(\mathbf{q}_{\|}+2 \pi i \mathbf{k} / a^{R}\right) \mathbf{n}_{\|} a^{L}\right) e_{\mathbf{k}, j \alpha}^{L}\right),
\end{array}
$$

And for the right side, in the form of superposition of the transmitted waves:

$$
u_{\mathbf{n}^{\prime}, \alpha}^{R}=\exp (i \omega t) \sum_{j, \mathbf{k}^{\prime}} B_{\mathbf{k}^{\prime}, j} \exp \left(i q_{j, x}^{L} n_{x}^{\prime} a^{R}+i\left(\mathbf{q}_{\|}+2 \pi i \mathbf{k}^{\prime} / a^{L}\right) \mathbf{n}^{\prime}{ }_{\|} a^{R}\right) e_{\mathbf{k}, j \alpha}^{L} .
$$

We substitute Eqs. (42), (43) into Eq. (8). Then, we divide the obtained equation by $\exp i\left(\mathbf{q}_{\|} \mathbf{n}_{\|} a^{L}\right)$ and multiply by $\left.\exp \left(2 \pi i \mathbf{l} / a^{R}\right) \mathbf{n}_{\|} a^{L}\right)$. Then, we sum over $\mathbf{n}$ so that the total number of additives would equal $N$, and divide by $N$.

We introduce:

$$
\begin{gathered}
K_{\mathbf{n}, \alpha \beta}\left(\mathbf{q}_{\|}\right)=\sum_{\mathbf{n}_{\|}^{\prime}, \beta} \frac{\partial^{2} U}{\partial u_{\mathbf{n}, \alpha}^{L} \partial u_{\mathbf{n}^{\prime}, \beta}^{R}} \exp \left(i \mathbf{q}_{\|}\left(\mathbf{n}_{\|}^{\prime} a^{R}-\mathbf{n}_{\|} a^{L}\right)\right), \\
K_{\mathbf{k} \mathbf{k}^{\prime}, \alpha \beta}^{L}\left(\mathbf{q}_{\|}\right)=\sum_{\mathbf{n}^{\prime} \mid \mathbf{n}_{\|, \beta}} \frac{\partial^{2} U}{\partial u_{\mathbf{n}, \alpha}^{L} \partial u_{\mathbf{n}^{\prime}, \beta}^{R}} \exp \left(i \mathbf{q}_{\|}\left(\mathbf{n}_{\|}^{\prime} a^{R}-\mathbf{n}_{\| \mid} a^{L}\right)\right) \exp \left(2 \pi i\left(\mathbf{k}_{\|} \mathbf{n}_{\| \mid} a^{L} / a^{R}+\mathbf{k}_{\|{ }^{\prime}} \mathbf{n}_{\|}^{\prime} a^{R} / a^{L}\right)\right) .
\end{gathered}
$$

Similarly, as was done in section 3 , we introduce the second dynamic matrices; the matrix describing the interaction of the waves with the same $\mathbf{q}_{\|}$:

$$
M_{\mathbf{k}, \alpha j}^{L, \pm}=\sum_{\beta}\left({ }^{\otimes} D_{\alpha \beta}\left( \pm \mathbf{q}_{j, x}^{L}+\mathbf{q}_{\|}+2 \pi i \mathbf{k} / a^{R}\right)-K_{0, \alpha \beta}\right) e_{\mathbf{k}, j \beta}^{L},
$$

the matrix describing the interaction with the oscillations on the opposite side of the interface:

$$
I_{\mathbf{k}, \alpha j}^{L}\left(\mathbf{q}_{\|}\right)=\sum_{\beta} K_{\mathbf{k k}^{\prime}, \alpha \beta}^{L}\left(\mathbf{q}_{\|}\right) e_{\mathbf{k}^{\prime}, j \beta}^{R} .
$$


and the matrix describing the interaction with the oscillations of the same crystal, but with another $\mathbf{q}_{\|}$:

$$
S_{\mathbf{k}, \alpha j}^{L, \pm}=\sum_{\beta} K_{\mathbf{k}, \alpha \beta} e_{\mathbf{k}, j \beta}^{L} .
$$

In these notations, we can finally write:

$$
M_{\mathbf{l}, \alpha j}^{L,-} A_{\mathbf{l}, j}+\sum_{\mathbf{k}} S_{\mathbf{k}, \alpha j}^{L} A_{\mathbf{l}-\mathbf{k}, j}+\sum_{\mathbf{k}^{\prime}} I_{\mathbf{k}^{\prime}, \alpha j}^{L}\left(\mathbf{q}_{||}\right) B_{\mathbf{k}^{\prime}, j}=-M_{\mathbf{0}, \alpha 1}^{L,+} \delta_{\mathbf{l 0}} .
$$

For the right crystal a similar approach gives:

$$
M_{\mathbf{1}^{\prime}, \alpha j}^{R,+} B_{\mathbf{l}, j}+\sum_{\mathbf{k}^{\prime}} S_{\mathbf{k}^{\prime}, \alpha j}^{R} B_{\mathbf{l}^{\prime}-\mathbf{k}^{\prime}, j}+\sum_{\mathbf{k}} I_{\mathbf{k}, \alpha j}^{R} A_{\mathbf{k}, j}=-I_{\mathbf{0}, \alpha 1}^{R} \delta_{\mathbf{l 0}} .
$$

Equations (57) and (58) taken together form a complete system of equations describing the transmission, reflection and scattering of phonons at the interface between two crystals.

Thus, it is seen that, on the one hand, even in the model of an ideal interface without defects and roughness, scattering due to the mismatch of the crystal lattices appears. Alternatively, the assumption in DMM that a phonon incident on the interface 'forget' its initial direction is, generally speaking, incorrect. The scattering reveals a quite determined structure; the wave vector of the transmitted wave, parallel to the interface, differs from the wave vector of the incident wave by an integer number of the vectors of the reciprocal lattice of the left crystal, while the wave vector, parallel to the interface, of the reflected wave differs from the wave vector of the incident wave by an integer number of the vectors of the reciprocal lattice of the right crystal. For vectors of the scattered phonons lying in the first Brillouin zone, all that remains is to rearrange them using Eq. (37).

It is impossible to solve the infinite system of Eqs. (57) and (58) analytically. However, if the function $\Phi$, in terms of which the matrix $K_{\mathbf{n}, \alpha \beta}$ is determined in Eq. (39), is rather smooth, the coefficients of its expansion into the Fourier series decreases rapidly, as do the matrices $K_{\mathbf{k}, \alpha \beta}$ describing the scattering. In this case, one can choose a finite number of the terms from the system $(57,58)$ and solve it numerically. In the case when the matrices $K_{\mathbf{k}, \alpha \beta}$ are large, even at large values of $\mathbf{k}$, one can consider the assumption of the DMM as being valid.

To facilitate the numerical calculation of the problem, it is reasonable to invoke the theorem on the matrix of the interfacial interaction in the general. Lets expand the function $\Phi$ into the Fourier series:

$$
\Phi(x)=\sum_{\mathbf{k}=-\infty}^{+\infty} \Phi_{k} \exp \left(2 \pi i \mathbf{k} x / a^{R}\right) .
$$

On substitution into Eq. (39), we find that the matrix $K_{\mathbf{k}, \alpha \beta}^{L}$ is expressed in terms of the components of $\Phi$ as:

$$
K_{\mathbf{k}, \alpha \beta}^{L}=k_{\alpha} \Phi_{k} .
$$

Thus, $K_{\left(0, k_{z}\right), y \beta}=0$ and $K_{\left(k_{y}, 0\right), z \beta}=0$. We use the fact that according to Eq. (23), $K_{\beta \alpha}^{R}=\left(a^{L} / a^{R}\right)^{2} K_{\alpha \beta}^{L}$ and that for $K_{\beta \alpha}^{R}$ the expression analogous to Eq. (39) is valid. We obtain:

$$
K_{0, \alpha \beta}^{L}=\beta \delta_{\alpha x} \delta_{\beta x}
$$

Thus, the atomic oscillations parallel to the interface do not contribute to the transmission of phonons without scattering at the interface.

\section{The uniform crystal limited case}

To test the system $(49,50)$, let us formaly apply it to the case of uniform crystal. We will formally name the plane $(1,0,0)$ in the crystal with simple cubic lattice as a boundary. Which means $a^{L}=a^{R}=a ; \beta_{1}^{L}=\beta_{1}^{R}=\beta_{1}$, $\beta_{2}^{L}=\beta_{2}^{R}=\beta_{2}$. Obviously enough, in this case, the phonons will not reflect at the 'interface' because there can be no reflection in a uniform medium so $\left|B_{1}\right|=1, A_{i}=B_{2}=B_{3}=0$. Let us check that it follows from the system $(49,50)$.

In the considered case, the matrix of interfacial interaction does not depend on the number of atoms:

$$
K_{\mathbf{n}, \alpha \beta}\left(\mathbf{q}_{\|}\right)=K_{\alpha \beta}\left(\mathbf{q}_{\|}\right)=\sum_{\mathbf{n}_{\|,}^{\prime}, \beta} \frac{\partial^{2} U}{\partial u_{\mathbf{n}, \alpha}^{L} \partial u_{\mathbf{n}^{\prime}, \beta}^{R}} \exp \left(i \mathbf{q}_{\|}\left(\mathbf{n}_{\|}^{\prime}-\mathbf{n}_{\|}\right) a\right),
$$

and all different matrices $K_{\mathbf{k k}^{\prime}, \alpha \beta}^{L}$ degenerate into a single matrix

$$
K_{\mathbf{k k}^{\prime}, \alpha \beta}^{L}=K_{\mathbf{k k}^{\prime}, \alpha \beta}^{R}=K_{\alpha \beta}\left(\mathbf{q}_{\|}\right)=\sum_{\mathbf{n}_{\| \mid}^{\prime} \mathbf{n}_{\|}, \beta} \frac{\partial^{2} U}{\partial u_{\mathbf{n}, \alpha}^{L} \partial u_{\mathbf{n}^{\prime}, \beta}^{R}} \exp \left(i \mathbf{q}_{\|}\left(\mathbf{n}_{\|}^{\prime}-\mathbf{n}_{\|}\right) a\right) .
$$




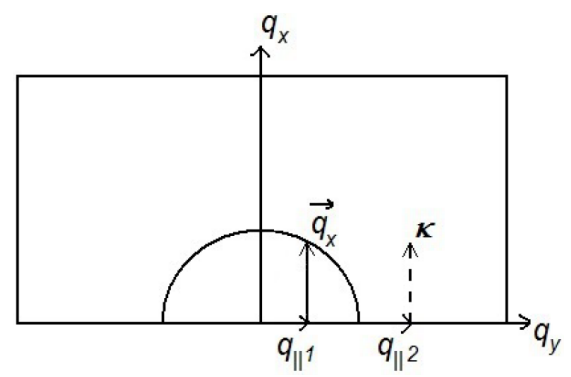

FIG. 2. A surface of constant frequency in the Brillouin zone. It is shown that a phonon with the component of the wave vector parallel to the interface, $q_{\| 1}$ passes through it. When an incident phonon has the component of the wave vector parallel to the interface $q_{\| 2}$, on the opposite side there appears an inward-damping oscillation with the damping coefficient $\kappa$ (shown by dashed arrow)

The second dynamical matrices also degenerate into a single one:

$$
M_{\alpha j}^{L, \pm}=\sum_{\beta}\left({ }^{\otimes} D_{\alpha \beta}\left( \pm \mathbf{q}_{j, x}^{L}+\mathbf{q}_{\|}\right)-K_{\alpha \beta}\right) e_{j \beta},
$$

the matrix describing the interaction with the oscillations on the opposite side of the interface:

$$
I_{\alpha j}^{L}\left(\mathbf{q}_{\|}\right)=\sum_{\beta} K_{\alpha \beta}\left(\mathbf{q}_{\|}\right) e_{j \beta},
$$

and

$$
S_{\mathbf{k}, \alpha j}^{L, \pm}=0
$$

After a similar transformation of the right crystal matrices, an infinite system of equations (49, 50) becomes a system of six equations:

$$
\begin{aligned}
& M_{\alpha j}^{L,-} A_{j}+I_{\alpha j}^{L} B_{j}=-M_{\alpha 1}^{L,+}, \\
& M_{\alpha j}^{R,+} B_{j}+I_{\alpha j}^{R} A_{j}=-I_{\alpha 1}^{R} .
\end{aligned}
$$

Let us verify that $B_{1}=\exp \left(i \mathbf{q}_{x} a\right), A_{i}=B_{2}=B_{3}=0$ is a solution. Indeed, after the substitution, we obtain:

$$
\begin{aligned}
I_{\alpha 1}^{L} \exp \left(i \mathbf{q}_{x} a\right) & =-M_{\alpha 1}^{L,+}, \\
M_{\alpha 1}^{R,+} \exp \left(i \mathbf{q}_{x} a\right) & =-I_{\alpha 1}^{R} .
\end{aligned}
$$

We substitude equations $(56,57)$ into the first equation of the system $(60)$ and obtain:

$$
\sum_{\beta} K_{\alpha \beta}\left(\mathbf{q}_{\|}\right) e_{1 \beta} \exp \left(i \mathbf{q}_{x} a\right)=-\sum_{\beta}\left({ }^{\otimes} D_{\alpha \beta}\left( \pm \mathbf{q}_{1, x}^{L}+\mathbf{q}_{\|}\right)-K_{\alpha \beta}\right) e_{1 \beta} .
$$

By defenition (55) $K_{\alpha \beta}\left(\mathbf{q}_{\|}\right)\left(1-\exp \left(i \mathbf{q}_{x} a\right)\right)={ }^{\otimes} D_{\alpha \beta}\left(\mathbf{q}_{j, x}^{L}+\mathbf{q}_{\|}\right)$, from which it follows that (61) holds identically. The second equation in the system is quite similarly checked (60).

\section{Refraction of phonons at the crystal interface}

Let's give a qualitative description of refraction of phonons at the crystal interface. To do so, we display the surface of the constant frequency $\omega$ in the Brillouin zone (Fig. 2) and make a projection of this surface onto the plane $\left(q_{y}, q_{z}\right)$. If the component $\mathbf{q}_{\|}$of the incident phonon is lying in the limits of this projection, it is easy to see that for the transmitted phonon the wave vector component normal to the interface, $q_{x}$, is equal to the $q_{x}$ of that point in the Brillouin zone which was projected into the point $\mathbf{q}_{\|}$.

If the component $\mathbf{q}_{\|}$of the incident phonon lies beyond the projection, the transmitted phonon does not appear, because there is no the $q_{x}$ such that the phonon with the wave vector $\left(q_{x}, q_{\| y}, q_{\| z}\right)$ has the frequency $\omega$. In this case, there occurs an oscillation directed into the depth from the interface, with the damping coefficient $\kappa$ and wave vector parallel to the interface $\mathbf{q}_{\|}$. The specific cases of such oscillations are well known Rayleigh waves [19]. Just as there are three branches of oscillations for the given $\omega, \mathbf{q}_{\|}$, one should perform the above procedure for each of them.

If the frequency of the incident wave exceeds the maximum possible in the given branch, in the crystal, an inward-damping oscillation at any value of $\mathbf{q}_{\|}$occurs. Oscillations of this kind were predicted in the model of a 
one-dimensional chain in paper [11]. Probably, it is such oscillations that were found in numerical simulations in work [16].

The maximum frequency of the transverse oscillations for a simple cubic lattice lies inside and not on the boundary of the Brillouin zone [17]. At some frequencies, the surface of constant frequency for these oscillation branches is closed (Fig. 3). In this case to every $\mathbf{q}_{\|}$, lying inside the projection of the isofrequency surface onto the plane $\left(q_{y}, q_{z}\right)$, correspond two values of $q_{x}$. To these two values of $q_{x}$ correspond different values of the derivative $\partial \omega / \partial q_{x}$, and, hence, different directions for energy propagation.

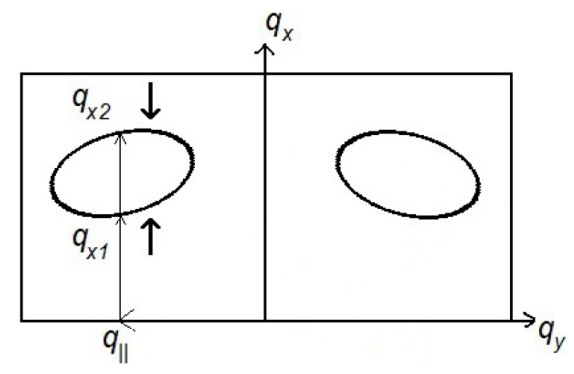

FIG. 3. It was shown that to each component $\mathbf{q}_{\|}$, lying inside the projection of the isofrequency surface onto the $\left(q_{y}, q_{z}\right)$, correspond two values of $q_{x}$. To these two values of $q_{x}$ correspond different values of the derivative $\partial \omega / \partial q_{x}$, and, hence, different directions for energy propagation (shown by solid arrows)

The transmitted waves should have the same sign $q_{x}$ as the incident (and the reflected the opposite) due to the principle of causality. In addition, because of the energy conservation law, the energy flux direction is the same for the incident and transmitted waves. If we assume that the incident wave is one in which the energy propagates towards the interface, then the value of $q_{x}$ in such a wave can be both positive and negative. Correspondingly, one should take the sign of $q_{x}$ in the transmitted and reflected waves. The uncertainty is thus eliminated.

An interesting effect occurs when the parallel-to-the interface component of the wave vector, $q_{\|}$, of the incident phonon is beyond the Brillouin zone of the right crystal (Fig. 4). In this case, the phonon is refracted and undergoes the Bragg scattering simultaneously. Then it appears that the refracted phonons have the opposite direction of propagation, in the plane of the interface, relative to the incident phonons. Thus, inverse refraction takes place.
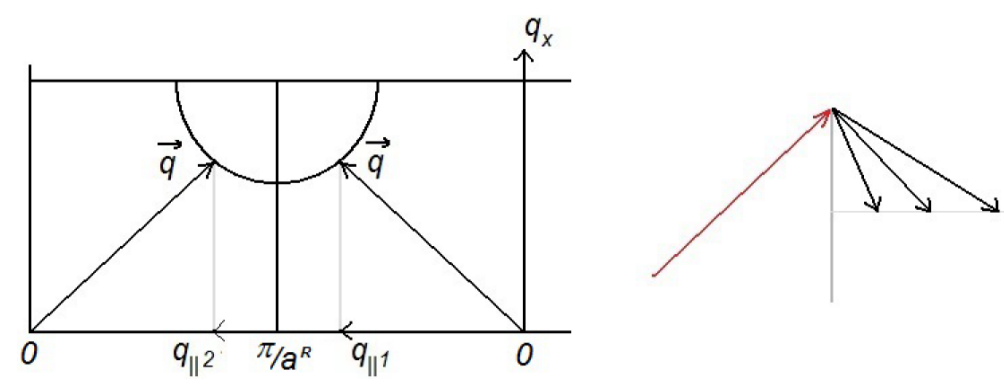

FIG. 4. Schematically displayed is the surface of the constant frequency in the Brillouin zone.

It was shown that the incident phonon with the parallel-to-interface wave vector component $q_{\|}$ lying outside the Brillouin zone of the right crystal, is refracted in the opposite direction

It is interesting to note, that the energy flux in the direction perpendicular to the interface should be conserved for each solution of the system of Eqs. (57), (58). However, for each solution, the energy flux in the plane of the interface can be different on the opposite sides from the interface. This means that at the interface not only the temperature may undergo a jump but the thermal flux parallel to the interface plane as well.

\section{Conclusion}

In this paper, we have studied the model of the interface between two crystals taking into consideration the mismatch of crystal lattices. The basic assumption of the model is that displacements of atoms near the interface are not random but determined by interaction with atoms of the other crystal. We have shown that in the harmonic 
approximation, such displacements have no effect on phonon transmission across the interface. The equation was set up, which determines the amplitudes of the transmitted and reflected waves of lattice oscillations. The exact solution has been derived for the quasi-one-dimensional case.

It was shown that the mismatch of lattices leads to the scattering of phonons at the crystal interface. In other words, the scattering appears even in the case of the ideal interface in the absence of defects and roughness. On the other hand, such scattering occurs non-uniformly in all directions but has a certain structure.

It has been established that the finiteness of the lattice parameter results, at certain angles of incidence, in the backward phonon refraction at the interface. A new family of lattice oscillations has been described, which includes only the atoms lying near the crystal interface. It was predicted that at the plane parallel to the interface, the heat flow experience a discontinuity at the interface.

The main result of our study is that we have shown that the oscillations of atoms in the plane of the interface interact weakly with the oscillations of atoms on the opposite side of the interface, except for specific resonance cases. In the case of normal incidence of phonons at the interface, the transmission coefficient of the transversely polarized phonons is much less as compared to that of the longitudinally polarized phonons. For an arbitrary angle of incidence, the transmission coefficients of phonons of any polarization are less than that calculated with the elasticity theory, even in the case of low frequencies. Allowance for this factor leads one to conclude that the calculated value of Kapitza resistance is approximately three times greater. Calculation of the interface thermal resistance, performed by means of the method proposed in the work [20], gives significantly lower values, as compared to those experimentally obtained. Accounting for the smallness of the transmission coefficient of transversely polarized phonons explains this discrepancy.

\section{Acknowledgements}

The author thanks E. D. Eidelman and G. V. Budkin for helpful critical comments, and A. Ya. Vul for attention to this study. The work was supported by the Russian Foundation for Basic Research (Project No. 16-03-01084 A).

\section{References}

[1] Kidalov S. V., Shakhov F. M. Thermal Conductivity of Diamond Composites. Materials, 2009, 2, P. $2467-2495$.

[2] Zhang C., Wang R., et al. Low-temperature densification of diamond/Cu composite prepared from dual-layer coated diamond particles. $J$. Material Science, 2015, 26, P. 185-190.

[3] Li B., Wang L., Casati G. Negative differential thermal resistance and thermal transistor. Appl. Phys. Lett., $2006, \mathbf{8 8}, 143501$.

[4] Terraneo M., Peyrard M., Casati G. Controlling the Energy Flow in Nonlinear Lattices: A Model for a Thermal Rectifier. Phys. Rev. Lett., 2002, 88, P. 094302.

[5] Wang L., Li B. Thermal Memory: A Storage of Phononic Information. Phys. Rev. Lett., 2008, 101, P. 267203.

[6] Khalatnikov I. M. Heat Transfer Between Solids and Liquid Helium II. JETP, 1952, 22, P. 687

[7] Little W. A. The Transport Of Heat Between Dissimilar Solids At Low Temperatures. Can. J. Phys., 1959, 37, P. $334-349$.

[8] Stoner R. J., Maris H.J. Kapitza conductance and heat flow between solids at temperatures from 50 to 300 K. Phys. Rev. B, 1993, 48, P. 16373.

[9] Swartz T., Pohl R. O. Thermal resistance at interfaces. Rev. Mod. Phys., 1989, 61, P. 605-608.

[10] Zhang L., Keblinski P., Wang J.-S., Li B. Interfacial thermal transport in atomic junctions. Phys. Rev. B, 2011,83, P. 064303.

[11] Meilakhs A.P., Eidelman E. D. New Model of Heat Transport across the MetalInsulator Interface by the Example of Boundaries in a DiamondMetal Composite. JETP Letters, 2013, 97, P. 38-40.

[12] Young D. A., Maris H. J. Lattice-dynamical calculation of the Kapitza resistance between fcc lattices. Phys. Rev. B, 1989, 40, P. 3685-3693.

[13] Hu M., Keblinski P., Schelling P. K. Kapitza conductance of siliconamorphous polyethylene interfaces by molecular dynamics simulations. Phys. Rev. B, 2009, 79, P. 104305.

[14] Tian Z., Esfarjani K., Chen G. Enhancing phonon transmission across a Si/Ge interface by atomic roughness: First-principles study with the Greens function method. Phys. Rev. B, 2012, 86, P. 235304.

[15] Saaskilahti K., Oksanen J., Tulkki J., Volz S. Role of anharmonic phonon scattering in the spectrally decomposed thermal conductance at planar interfaces. Phys. Rev. B, 2014, 90, P. 134312.

[16] Yang N., Luo T. et. al. Thermal Interface Conductance between Aluminum and Silicon by Molecular Dynamics Simulations. J. Comp. and Theor. Nanoscience, 2015, 12, P. 168.

[17] Anselm A. I. Introduction to Semiconductor Theory. Englewood Cliffs.: Prentice-Hall, 1981.

[18] Arnold V. I. Collected Works, Vol. 1, Springer, 2009, 253 p.

[19] Landau L. D., Lifshitz E. M. Course of Theoretical Physics, Vol. 7: Theory of Elasticity. New York.: Pergamon, 1986.

[20] Meilakhs A.P. Nonequilibrium Distribution Function in the Presence of a Heat Flux at the Interface between Two Crystals. Phys. Solid State, 2015, 57, P. 148-152. 